\title{
Corrigendum: Tether: A Study on Bubble-Networks
}

\author{
Giovanni Rosa and Remo Pareschi * \\ Stake Lab, University of Molise, Campobasso, Italy \\ Keywords: tether, blockchain, stablecoin, social network analysis, transaction graph, bubble effect
}

\section{A Corrigendum on}

Tether: A Study on Bubble-Networks

by Rosa, G., and Pareschi, R. (2021). Front. Blockchain 4:686484. doi:10.3389/fbloc.2021.686484

In the original article, there were 2 errors. I) The term "Smalltalk" was erroneously used instead of "Smallworld" in the abstract; II) The term "small-talk" was erroneously used instead of "small-world" in the first paragraph of Section 6 Conclusion.

Tether is a stablecoin, namely a cryptocurrency associated with an underlying security. Tether provides one of the most relevant ways to buy bitcoins and has been the centre of many controversies. In fact, it has been hypothesized that new tethers are issued without the underlying reserves, and that new massive Tether emissions are the basis of strong speculative movements on the Bitcoin, with consequent bubble effects. In the course of this article, we conduct a Social Network Analysis focused on the Tether transaction graph to identify the main actors that play a leading role on the network and characterize the transaction flow between them. From our analysis, we conclude that 1) the Tether transaction network does not enjoy the "Smallworld" property, with the robustness and reliability it carries with it; 2) cryptopcurrency exchanges are the nodes with the greatest centrality; 3 ) even Assortativity is not found, as the subjects who move Tether on a large scale do not give continuity to their presence and operations, therefore do not get a chance to consolidate stable links between them; and 4) among the exchanges, Bitfinex, which has co-ownership and co-administration relationships with the Tether issuer, can be mostly associated with the Rich-gets-Richer property.

Summing up, the following indications can be drawn from our analysis of the Tether network: 1) this network (unlike that of Bitcoin) does not enjoy the "small-world" property with its characteristic traits of robustness and reliability; 2) the nodes with greater centrality are all exchanges, thus adding evidence to the hypothesis of the merely financial and supposedly speculative use of Tether; 3) on the other hand, this is not enough to create an effect of assortativity binding these nodes together, apparently due to the temporal transience of their role which bars the development of long-lasting relationships, since exchanges that are particularly active in a phase of heavy Tether traffic may be replaced at a later stage by other exchanges; 4) to these, however, Bitfinex is an exception, as it maintains a central role over times. These indications, taken together with the shareholding relationship between Bitfinex and the Tether issuer, are circumstantial elements in favor of a significant role played by Bitfinex in the maneuvers on the Tether that might influence the trend in Bitcoin prices.

The authors apologize for this error and state that this does not change the scientific conclusions of the article in any way. The original article has been updated. 
Publisher's Note: All claims expressed in this article are solely those of the authors and do not necessarily represent those of their affiliated organizations, or those of the publisher, the editors and the reviewers. Any product that may be evaluated in this article, or claim that may be made by its manufacturer, is not guaranteed or endorsed by the publisher.
Copyright $\odot 2022$ Rosa and Pareschi. This is an open-access article distributed under the terms of the Creative Commons Attribution License (CC BY). The use, distribution or reproduction in other forums is permitted, provided the original author(s) and the copyright owner(s) are credited and that the original publication in this journal is cited, in accordance with accepted academic practice. No use, distribution or reproduction is permitted which does not comply with these terms. 